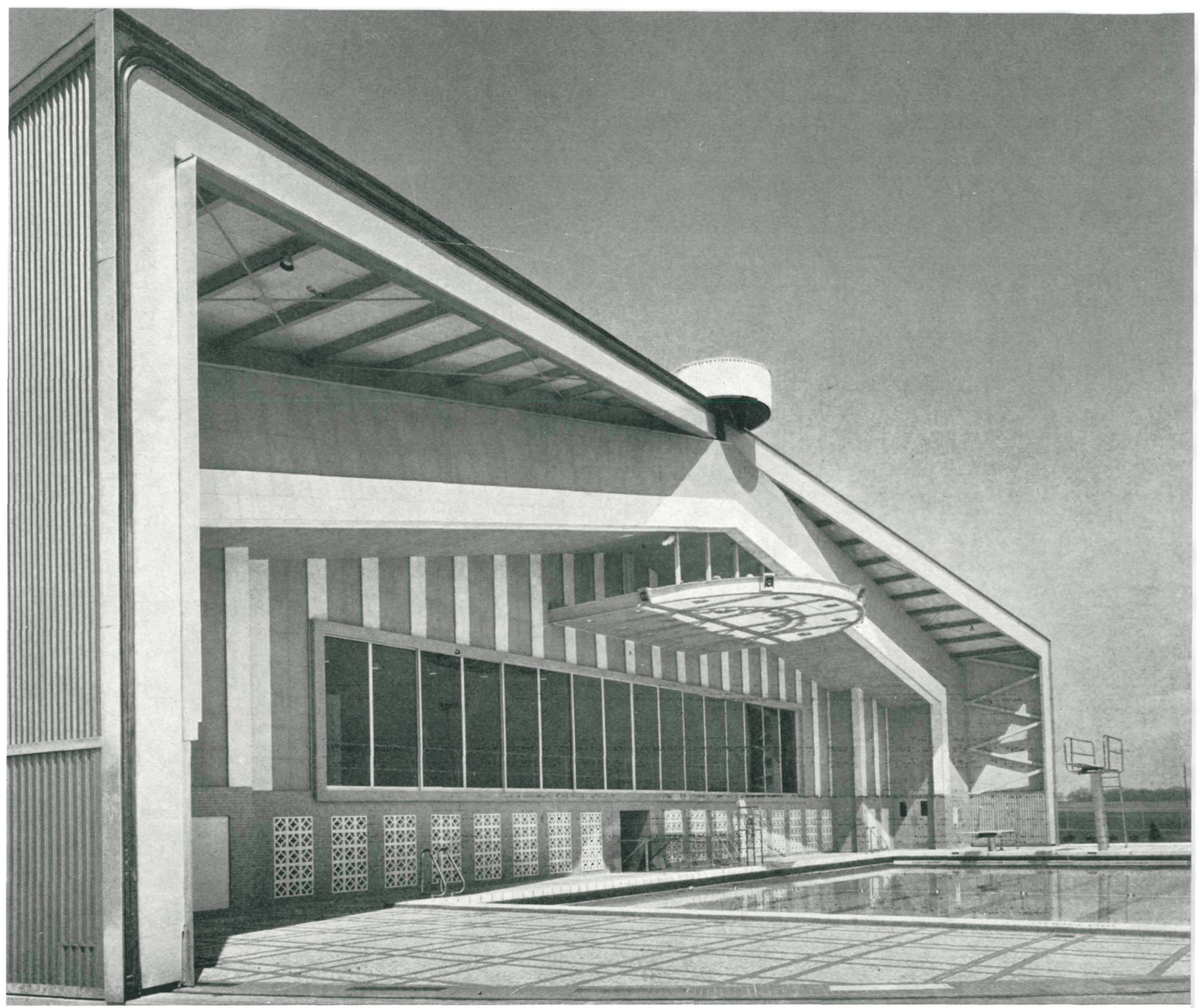

\title{
cubierta móvil para piscina, en Pensilvania
}

LAWRIE \& GREEN, arquitectos

Sinopsis Esta piscina, que forma parte de un centro recreativo en Hershey, Pensilvania, propiedad de Cocoa tento, permite dejar dicha piscina al aire libre en verano y poder cerrarla en invierno. El sistema está constituido por dos sectores, que cubren en planta un semicírculo, apoyados sobre patines de ruedas que corren a lo largo de una vía circular.

La estructura es metálica, a base de cerchas radiales que concurren en un muñón, el cual permite el giro de los sectores.

Las fachadas y cubiertas se han cerrado con bloques de espuma de vidrio, ligeros, impermeables y aislantes, cuyas características más destacadas son las siguientes: resistencia $\mathrm{de} 7 \mathrm{~kg} / \mathrm{cm}^{2}, 144 \mathrm{~kg} / \mathrm{m}^{3} \mathrm{de}$

$886-13$ peso y su gran capacidad de aislamientoadas son las siguientes: 


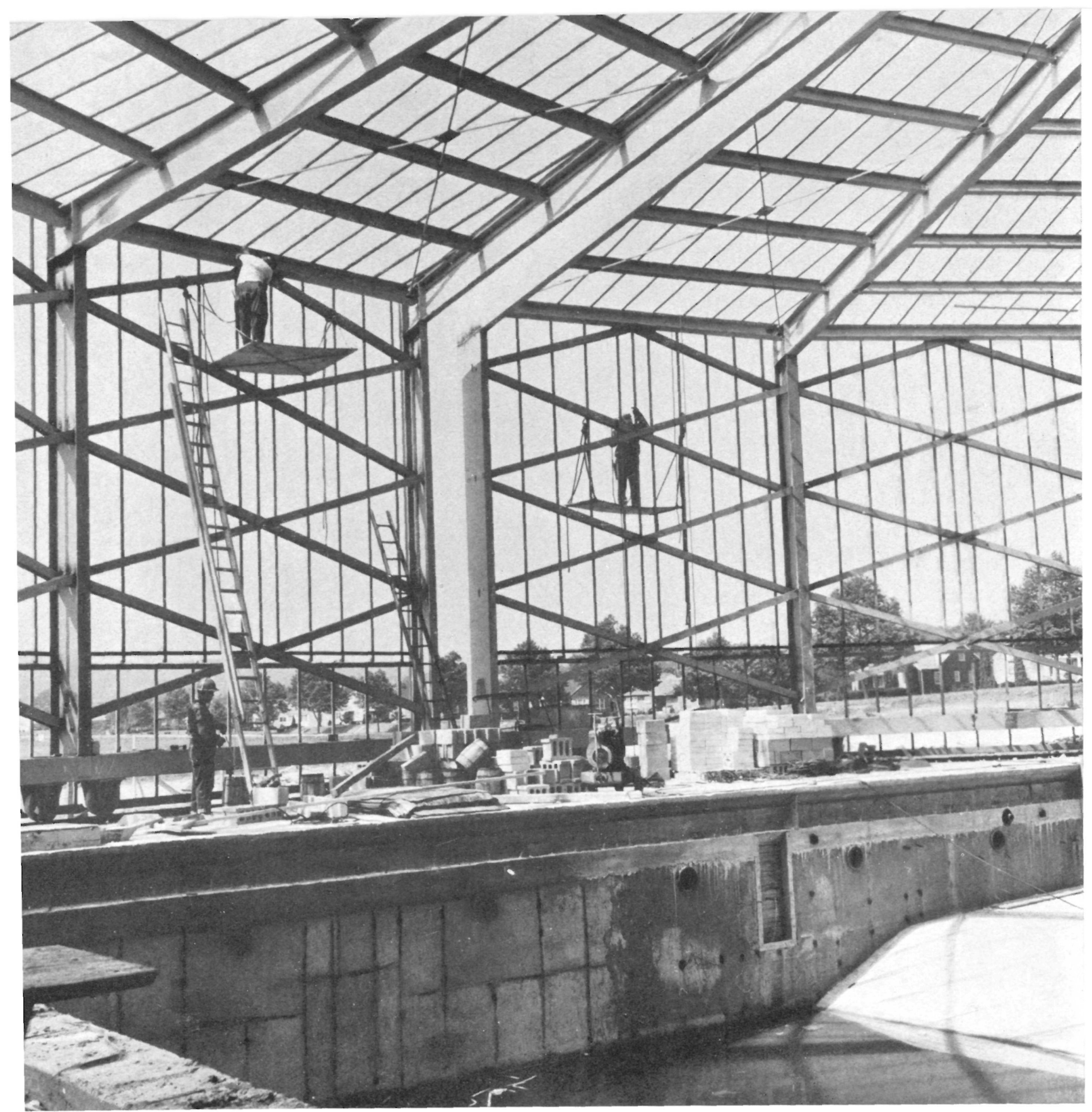

Entramado metálico.

El nuevo centro de recreos denominado Cocoa Avenue Plaza, en Hershey, estado de Pensilvania, EE. UU. de reciente construcción, tiene una piscina cuya cubierta, móvil, puede girar apoyándose en un muñón - montado en la parte superior y sobre una vía circular en el suelo-, que permite cerrar y cubrir la piscina en la temporada invernal y dejarla al aire libre durante el período estival.

La estructura, que es metálica y está formada por una serie de cerchas radiales, tiene un desarrollo

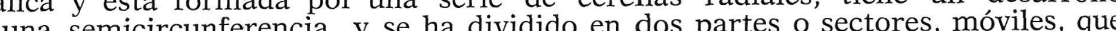
por corrimiento pueden ocupar dos posiciones diametralmente opuestas: en una de ellas la piscina se encuentra cubierta, mientras que en la otra se halla a cielo abierto. 
1. Conducto de aire de los vestuarios.2. Vestibulo.-3. Tragaluz.-4. Almacén.5. Tuberías. -6. Empleados -7. Cabina.8. Paso.-9. Instalaciones.-10. Junta en la solera.-11. Placas de aluminio.-12. Parte móvil.-13. Cerramiento de aluminio.-14. Toma para filtros.-15. Agua fría.-16.
Azulejo.-17. Nivel del agua.-18. Desagüe.

Todas las cerchas tienen forma de $\mathrm{L}$ y convergen hacia un muñón montado sobre un pórtico fijo y por encima de la parte central Estas cerchas, debidamente arriostradas entre ellas, forman dos sectores independientes que pueden girar apoyándose en el muñón superior y en la vía circular que descansa sobre el suelo. La parte inferior de cada cercha lleva montados una serie de patines de dos ruedas con pestaña que rodando sobre el raíl permiten correr, circularmente, el sector.

El arriostramiento entre cerchas en la parte superior se ha realizado con perfiles laminados de sección transversal en forma de doble T, y con pares de barras que pueden tesarse. El arriostramiento lateral constituye el entramado de cierre. Las piezas transversales, de mayor sección, forman un reticulado de malla romboidal, mientras que las verticales, mucho más ligeras, sirven de soporte a los bloques aislantes de cierre, de espuma de vidrio Foamglas, fabricados por la casa Pittsburgh Plate Glass Company, que presentan la doble ventaja de ser ligeros y sólidos, ya que pueden resistir hasta

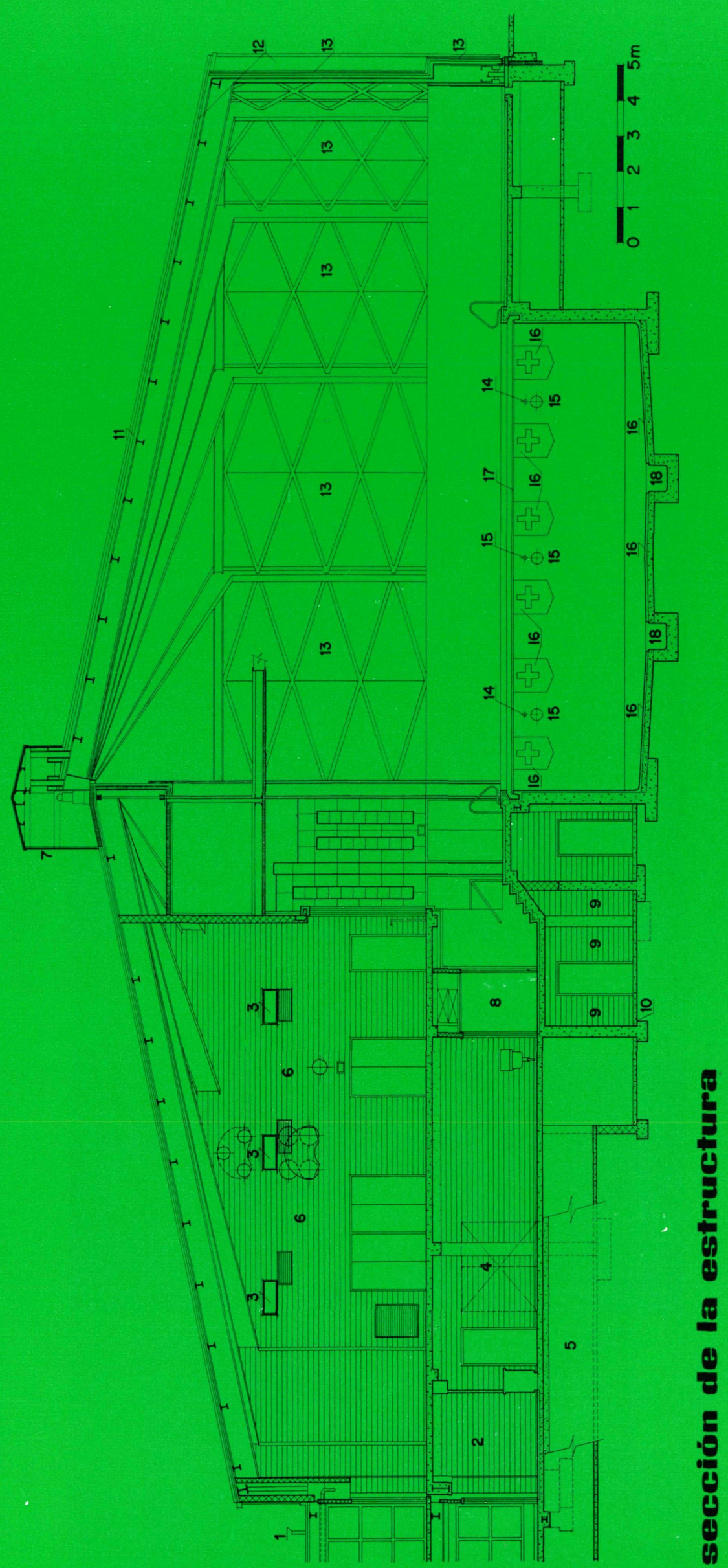



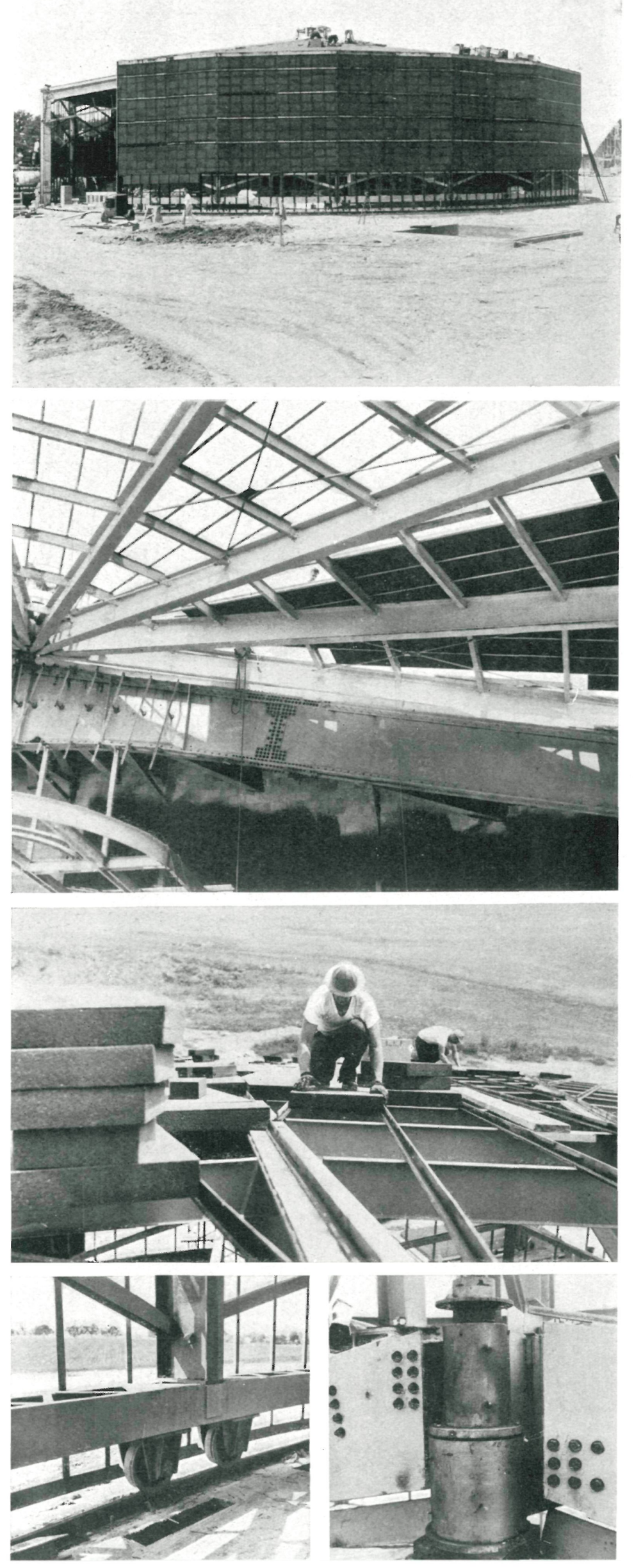

Construcción de muros exteriores.

Cerchas de cubierta.

Colocación de bloques de cierre.

Patín de ruedas y muñón.

Piscina abierta.

$7 \mathrm{~kg} / \mathrm{cm}^{2}$, se comportan bien frente a la humedad y vapor acuoso y tienen características que han permitido, a los arquitectos autores del proyecto, el empleo de una estructura ligera, dado que los citados bloques de cerramiento de fachadas y $\mathrm{cu}-$ biertas son de reducido peso propio $\left(144 \mathrm{~kg} / \mathrm{m}^{3}\right)$.

Los muros exteriores consisten en dos capas de bloques de espuma de vidrio, la primera de $10 \mathrm{~cm}$ de espesor y la segunda de $5 \mathrm{~cm}$, pegadas con asfalto caliente. E1 revestimiento exterior se ha realizado con paneles de aluminio pintados de blanco y azul, que dan un aspecto atractivo a las fachadas. En el interior, los paneles de aluminio llegan sólo hasta una altura de $1,80 \mathrm{~m}$ a partir del suelo. El resto de los muros e intradós de la cubierta llevan pintura blanca a base de vinilo.

La cubierta tiene forma de semicircunferencia, en la que las cerchas, radiales, convergen en el muñón central. Dichas cerchas son, en realidad, vigas de doble $\mathrm{T}$, de gran canto $\mathrm{y}$ alma llena, entre las que se han colocado las correas-laminados con sección de doble $\mathrm{T}$-, en donde se asientan los cabios. Estos están constituidos por laminados de sección en forma de $\mathrm{T}$ y colocados con las alas hacia abajo para que sirvan de apoyo a los bloques de espuma de vidrio que cierran la cubierta. Dichos bloques, que tienen $61 \times 45 \times 10 \mathrm{~cm}$, son celulares y se han fijado con una cinta, adhesiva por sus dos caras, fabricada por Pittsburgh Plate Glass Company y colocada sobre las alas de los cabios. 


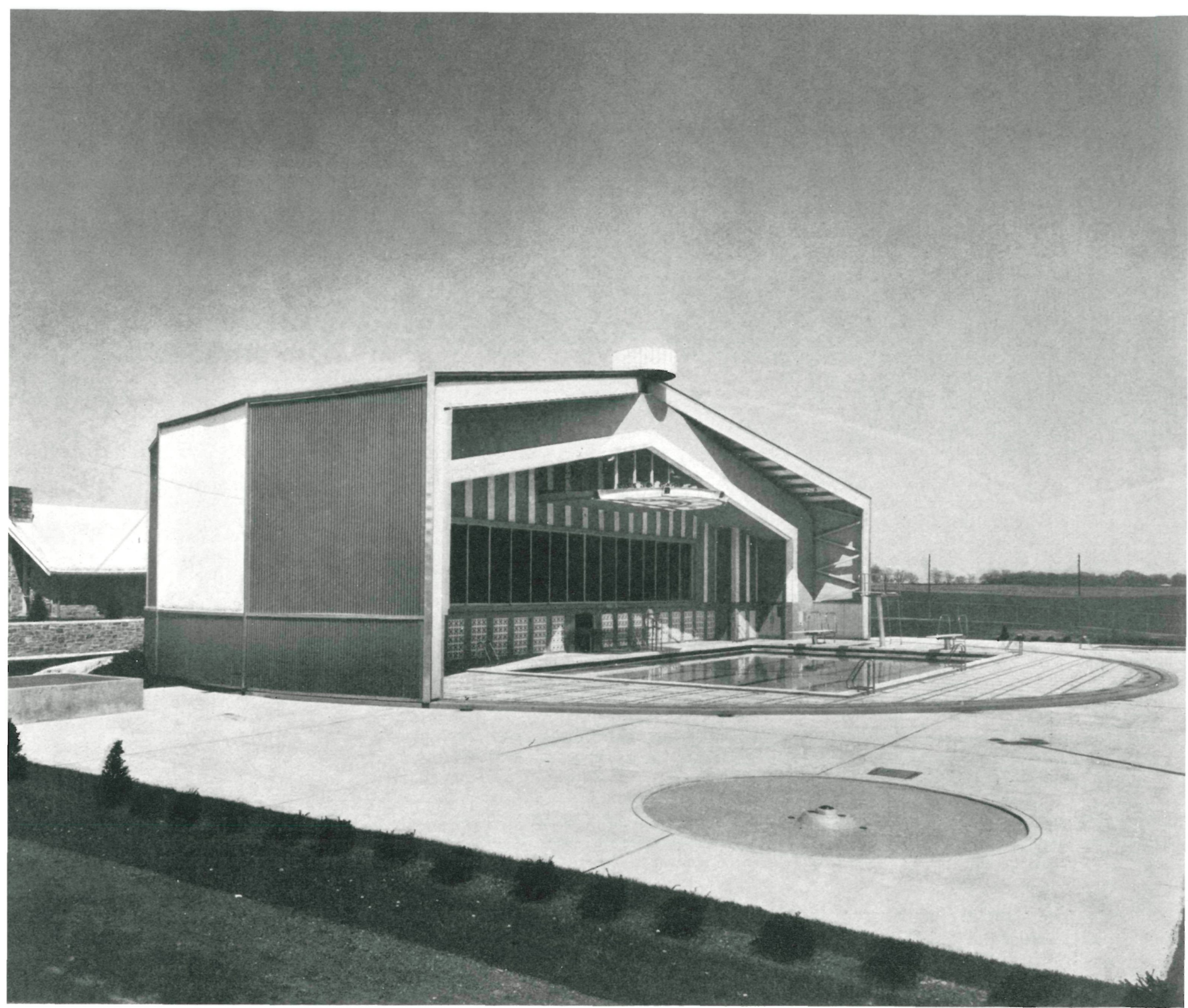

Fotos: LAWRENCES S. WILLIAMS, INC.

Los bloques de cierre de la cubierta se pueden cortar con una sierra de mano, lo que permite ajustar las piezas irregulares a las dimensiones y forma de los huecos que dejan las hileras de bloques de dimensiones ordinarias. Después de colocar la primera capa se procedió a tender la segunda, extendiendo antes un embadurnado de asfalto caliente, y escaqueando las juntas de los bloques de esta última respecto de los de la primera. Para terminar se colocó una capa de áridos blancos liados con un aglomerante.

Con objeto de impermeabilizar las juntas formadas entre los sectores móviles de la cubierta, se ha colocado un tubo que puede hincharse para asegurar un cierre perfecto.

Traducido y adaptado por J. J. Ugarte. 


\section{Couverture moloile pour piscime à Penmsglvamie}

Lawrie \& Green, architectes

Cette piscine, qui fait partie d'un centre récréatif à Hershey, Pennsylvanie, propriété de Cocoa Avenue Plaza, présente la curieuse particularité de sa couverture mobile qui permet de laisser, en été, la piscine à l'air libre et de la couvrir en hiver. Le système est constitué par deux parties mobiles qui couvrent, en plan, un demi-cercle, appuyées sur des roues qui roulent le long d'un rail circulaire.

La structure est métallique, réalisée à l'aide de cintres radiaux qui, partant d'un pivot, permettent la rotation des deux parties.

Les façades et les couvertures ont été réalisées à l'aide de blocs en "foamglass", légers, imperméables et isolants, dont les caractéristiques les plus importantes sont les suivantes: résistance de $7 \mathrm{~kg} / \mathrm{cm}^{2}, 144 \mathrm{~kg} / \mathrm{m}^{3}$ de poids et leur grande capacité d'isolation et d'imperméabilisation.

\section{Mobile poof swimming pool, in Pemmsilucumia}

Lawrie \& Green, arcihtects

This swimming pool constitutes part of a recreation centre at Hershey, Pennsilvania, and has the special feature that its roof is movable, so that the pool can be open in summer and enclosed in winter. The roof consists of two semicircular sectors, resting on wheels, which run along a circular rail.

The structure of this roof is metallic, made of radial trusses, converging at a central pivot, around which the sectors rotate.

Both the enclosing walls, and the roofing units are filled in with foamglass blocks, which are light, waterproof and highly insulating. Their more significant characteristics are a strangth of $7 \mathrm{~kg} / \mathrm{cm}^{2}$ and $144 \mathrm{~kg} / \mathrm{m}^{3}$ weight, as well as their small water and heat transmission capacity.

\section{Beweglichess Vemedeck film Schwimmulocud, in Pennsglvanien}

Lawrie \& Green, Architekte

Dieses Schwimmbad, welches zu einem Erholungszentrum in Hershey, Pennsylvanien, gehört und Eigentum der Cocoa Avenue Plaza ist, zeichnet sich durch die Eigentümlichkeit seines Verdeckes aus, das beweglich ist und daher gestattet, das Schwimmbad im Sommer offen und im Winter geschlossen zu betreiben. Das System besteht aus zwei Sektoren, welche auf der Grundfläche einen Halbkreis bilden und auf Gleiträdern ruhen, die in einer kreisförmigen Bahn laufen.

Die Metallstruktur besteht aus radialen Bogenrippen, die in einem Zapfen zusammenlaufen, der die Drehung der Sektoren gestattet.

Die Aussenseiten und Verdecke sind mit leichten, wasserdichten und isolierenden Glasschaumblöcken abgedeckt, deren wichtigste Charakteristiken folgende sind: Festigkeit $7 \mathrm{~kg} / \mathrm{cm}^{2}$, Gewicht $144 \mathrm{~kg} / \mathrm{m}^{3}$ und grosse Isolierfähigkeit und Wasserdichtheit. 